\title{
Self-Reflection Control of Mental States During Academic Activity
}

\author{
Aleksandr Oktyabrinovich Prokhorov
}

Kazan (Volga Region) Federal University, Russia, 420008, Kazan, Mezhlayk Street, 1

\section{Albert Valentinovich Chernov}

Kazan (Volga Region) Federal University, Russia, 420008, Kazan, Mezhlayk Street, 1

\section{Doi:10.5901/mjss.2015.v6n3s2p277}

\section{Abstract}

The article is devoted to the patterns of self-reflection's influence upon the mental states graded according to various criteria: modality, duration, sign, and level of arousal. It has been fixed that the influence of self-reflection upon the mental states was mediated by the cognitive and controlling components of self-reflection, and also connected both to the academic activity types (a lecture, seminar, exam) and students' field of study.

Keywords: self-reflection, mental states, academic activity type, controlling features, field of study.

\section{Introduction}

The self-reflection, as a component of controlling processes, is the least understood in course of exploration of the principal determining mechanisms concerning various mental states: contextual, cognitive, connotative, personal, etc. It is necessary to point out in this very context that the academic activity imposes sufficient requirements to the selfreflection's level of development, while the successful learning of new knowledge by the students, and also behavioral and studying efficient self-control greatly depend on this very self-reflection's involvement into the academic process.

After A.V. Karpov, the self-reflection could be determined as "both the unique property intrinsic to the human-being only on the one hand, and also the state of selfconsciousness of anything on the other hand, and finally, the representation process of its own content to the human psychic as well; however, could be never come down to one of the above mentioned aspects" [1, p. 86]. While researching the self-reflection as a mental process, the author states that the self-reflection serves as the multi-process phenomenon synthesizing in it the whole variety of cognitive, controlling and communication processes.

There is no agreement in researchers' opinion as concerning the essence of self-reflection. Thus, the self-reflection is treated as a susceptibility to self-analysis and most often determined as both an ability and susceptibility to think about oneself and other persons as subjects influenced by inner states [2,3]. Furthermore, the self-reflection is also associated with meta-cognitions implied to be the knowledge about oneself as a perceptive subject and the control of one's own cognition [4]. Another approach consists in correlation of self-reflection to ideas of mind. This very position presupposes the statement that some mental states differ from external events and actions $[5,6]$. Finally, another theory treats the selfreflection as the self-consciousness and so, presupposes the thinking over the personal experience [7].

The interconnection between the self-reflection and mental states in the context of self-control as for these very mental states is of special importance. The self-reflection is "involved" in the controlling process of the subject as a central core, self-determining and self-controlling framework of its control actions [8]. Due to self-reflection, the selfconsciousness, assessment and collation of the current state to the desired one are performed. Moreover, in case of necessity, the subject makes some correction of the applied ways and methods of control.

\section{Methods of Research}

The influence of self-reflection upon the mental states of the students was explored on the basis of two survey samples: 1) 73 third-year engineering students (31 men and 42 women); 2) 75 second- and third-year students of the humanities (18 men, 57 women). The age of those surveyed was 18 to 21 years old. The research was performed in three various kinds of academic activity: a lecture, seminar, exam (concerning vocation-related subjects corresponding to the chosen 
field of study).

Both the lecture and seminar are marked by low level of tension. That is why; the students' states were treated as common, everyday. On the contrary, at the exam these very states are supposed to be "stressful", due to the great significance and tension of the latter academic activity. The experiment was performed in the middle of the class simultaneously, when the students were actively involved into the studying process. The lecture was interrupted in twenty minutes after beginning for the exploration procedures. During the seminars the test was being fulfilled in course of report's discussion dedicated to the class topic. The exploration at the exam was performed just after receiving the tasks. The students were offered to put aside the exam card and take part in the research.

Towards revealing the peculiarities of the self-reflection's influencing upon the mental states the "polar group" technique was applied: the whole unity of the surveyed students were subdivided into three groups according to selfreflection expressiveness.

The obtained data were processed using the multivariate analysis of variance (MANOVA) that is a component of SPSS 16.0 statistical set. Moreover, the statistic of comparison and connection ( $\mathrm{t}$ - Student's criterion) and Pearson product-moment correlation coefficient were also used during the data analysis.

The estimation of structural integrity and differentiation rate was accomplished using A.V. Karpov's technique. It allows to calculate the structure orderliness index (SOI) [1].

The following methods and techniques were applied in course of research performance:

1. A.V. Karpov and V.V. Ponomareva's Self-Reflection Development Level Diagnostic Technique [9]. 2. M. Grant's Self-Reflection Aspects Measurement Technique allowing to estimate the expressiveness and orientation of self-reflection [10]. 3. V.I. Morsanova's Method "Behavioral Self-Regulation Style" [11]. Metacognitive Activity Involvement Diagnostic Technique (MAl) by Schraw \& Dennison [12]. 5. Method "Behavioral Self-Regulation Style" [13]. 6. Checklist "Personality Mental State Relief" [14].

\section{Main Points}

In course of the obtained results processing by means of the multivariate analysis of variance (MANOVA) it was fixed that the self-reflection influences upon the mental states usual for the academic activity, which are classified according to duration: short-term and long-term ( $p=0.022$; Fisher's criterion $=3.875)$; level of arousal: low, medium and high intensity $(p=0.026$, Fisher's criterion $=2.800)$; also, sign: positive and negative $(p=0.002$, Fisher's criterion $=6.452)$.

The impact of self-reflection on the students in everyday academic activity. The following mental states were revealed in the everyday academic activity: emotional (joy, calmness), volitional (resoluteness, readiness) and intellectual (dreaminess, commitment). The research proved that the states of commitment and calmness are the most typical mental states felt by the students during the everyday academic activity (at the lecture and seminar). From now forward, these very two mental states will be considered.

The findings states that the sufficient influence upon the state of commitment makes auto self-reflection $(p<0.05$; Fisher's criterion $=1.450$ ). Moreover, the students experience this mental state the most intensively in case of medium auto self-reflection. The key indicator influencing upon the state of calmness is the control self-reflection, the metacognitive involvement into activity, in particular $(p<0.006$, Fisher's criterion $=5.800)$.

Towards revealing the impact degree of the self-reflection on the various substructures of mental states, the structure orderliness index (SOI) made by the interaction between the self-reflection parameters and typical states, i.e., commitment and calmness was calculated (Table 1).

Table 1. The Structure Orderliness Index for Commitment and Calmness

\begin{tabular}{|c|c|c|}
\hline Mental state's constituents / SOI & Commitment & Calmness \\
\hline Mental processes & 4 & 3 \\
\hline Physiological responses & 0 & 0 \\
\hline Experiences & 1 & 4 \\
\hline Behavior & 5 & 13 \\
\hline Total & 10 & 20 \\
\hline
\end{tabular}

As we can see from Table 1, the state of calmness demonstrates higher SOI than commitment. It is caused by numerous interrelations between various parameters of self-reflection and behavioral characteristics usual for calmness. In both cases the behavioral characteristics are leading within the mental states' structure. It means, either increase, or decrease 
in the self-reflection's indicators will first of all affect the activity and consistency of the subject's behavior. The mental states usual for everyday academic activity are intensively felt in case of medium cognitive and high control selfreflection.

The impact of self-reflection on the students in stressful academic activity (exam). The stressful situation of the exam stated mainly negative emotional and volitional states. As follows from the research's findings, "concentration" and "despondency" are the most typical states for the exam. The above mentioned states are registered for the students in $53 \%$ cases at the exam. On that premise, these very two mental states will be considered from now forward.

All students surveyed were divided into groups according to the self-reflection intensity. It was stated that number of individuals experiencing the low level of arousal increases together with self-reflection's degree raises. Referring to Fig. 1, about $45 \%$ of "high-reflecting" students experience the low intensity states, whereas this rate is rather lower for "low-reflecting" ones - only $28 \%$. The latter gives evidences that the "low-reflecting" students easier rub through the stressful situation, while they do not usually analyze their inner experiences as for the event, and so, avoid "sinking" into negative states. The "low-reflecting" ones also demonstrate the highest rate of the individuals experiencing the state of increased level of arousal (about 18\%). This indicator steadily declines together with the raise of the self-reflection's characteristics.

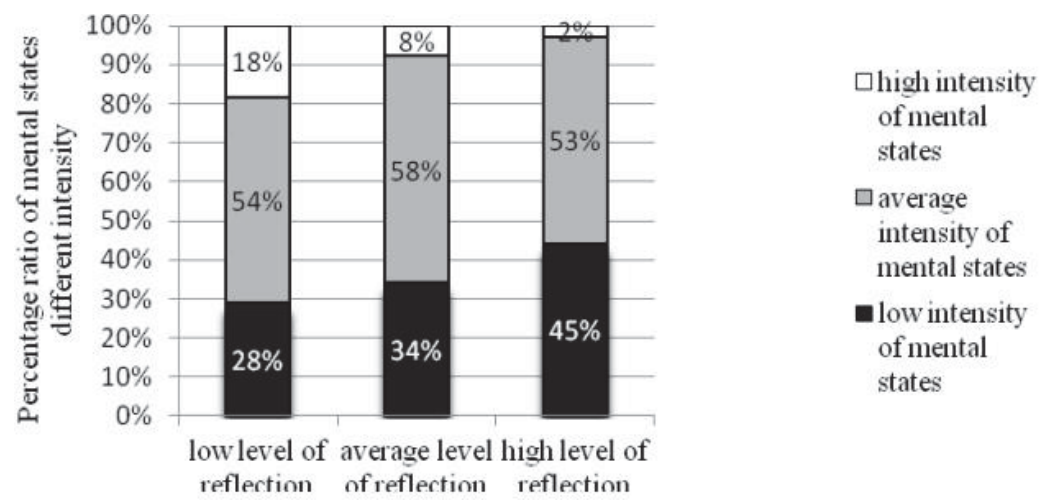

Fig. 1. The Correlation of Mental States' Intensity at the Exam for Groups of Students with Various Self-Reflection's Levels

The exploration's results report that the medium self-reflection makes an impact on the intensity increase for the low activity mental states in the stressful situation, and causes the decrease in the high activity states at the same time. The state of despondency experienced by the "low-" and "high-reflecting" students are marked by extremely low characteristics of intensity, as the former reflexes the experienced state to a smaller extent, and so, never control it, and the latter give all their attention to experiencing of this very state and sink in it deeply. According to our reckoning, it is caused by the fact that the self-control process directed to one area of activity declines self-control in another one, as it was proven in the explorations of E.A. Sergiyenko [15]. The mental states of high intensity are slightly reflexed by the "low-reflecting" students, and consequently, are controlled to a little extent.

The substructure orderliness indexes (SOI) for concentration and despondency of the students in the situation of the exam are shown in Table 2. It should be noted that the state of "despondency" is more connected to cognitive and control parameters of the self-reflection than "concentration". The higher structure orderliness index proves that demonstrably.

Table 2. The Structure Orderliness Index for Despondency and Concentration

\begin{tabular}{|c|c|c|}
\hline Mental state's constituents / SOI & Despondency & Concentration \\
\hline Mental processes & 0 & 0 \\
\hline Physiological responses & 8 & 3 \\
\hline Experiences & 8 & 2 \\
\hline Behavior & 4 & 3 \\
\hline Total & 20 & 8 \\
\hline
\end{tabular}


The influence of both the auto self-reflection's parameters and academic situations (everyday along with stressful) upon the intensity of mental states depending on the qualitative characteristics of the latter was reported in course of the exploration. The results obtained involving the analysis of variance possess a great level of significance $(p<0.001)$. The very influence of the auto self-reflection and qualitative characteristics never get a great confidence level, however, the interaction of these variables is significant at $p<0.003$ depending on the situation.

Towards revealing the aggregated influence of the self-reflection, subject's control abilities, types of academic activity and field of study upon the substructures of mental states the analysis of variance was applied. The chosen model is statistically significant at the rate of $p<0.001$ and is suitable for explanation of $34 \%$ of average values dispersion of the mental states (Table 3). The influence of the types of academic activity $(p<0.001)$, field of study $(p<0.017)$, level of control abilities $(p<0.001)$, and also self-reflection of activity $(p<0.05)$ are statistically significant (according to Fisher's criterion). It should be noted that the interaction of such variables, as "self-reflection" and kinds of academic activity $(p<0.004)$, and also self-reflection with the field of study $(p<0.026)$ result in the sufficient influence upon the intensity of the states experienced by the students.

Table 3. The Impact of Self-Reflection on Mental States

\begin{tabular}{|c|c|c|c|c|c|}
\hline \multicolumn{7}{|c|}{ Dependant variable: Mental states (structurally average values) } \\
\hline Source of variation & Quadratic sum & $d f$ & Mean square & Fisher's criterion & P-rate \\
\hline Model & 288.804 & 52 & 5.553 & 2.130 & .000 \\
\hline AAT & 39.944 & 2 & 19.972 & 7.660 & .001 \\
\hline Field of study & 15.038 & 1 & 15.038 & 5.767 & .017 \\
\hline CAL & 35.344 & 2 & 17.672 & 6.77 & .001 \\
\hline SRA & 15.305 & 2 & 7.652 & 2.93 & .050 \\
\hline AAT * SRA & 41.965 & 4 & 10.491 & 4.024 & .004 \\
\hline Field of study ${ }^{*}$ SRA & 19.358 & 2 & 9.679 & 3.712 & .026 \\
\hline CAL ${ }^{*}$ SRA & 47.803 & 4 & 11.950 & 4.478 & .002 \\
\hline \multicolumn{7}{|c|}{ Determination factor $R^{2}=0.336$} & & \\
\hline
\end{tabular}

Legend: $\boldsymbol{A A T}$ - academic activity type; SRA - self-reflection of activity; CAL - level of control abilities; * ${ }^{*}$ variables reciprocity factor

The unexplained part of dispersion could be a result of the influence upon the mental states of the various personal traits, because the sufficient number of interconnections within the mental states' functional structure accounts for these traits exactly [16]. The obtained findings indicate that the connection between mental states' parameters and the self-reflection is complicated and mediated by both the field of study and the type of academic activity. This is the manifestation of the self-reflection's transformational function.

The impact of self-reflection's level on the mental states depending upon the field of study. The states with the highest intensity are experienced by the "low-reflecting" students of the technical sciences and "medium-reflecting" students of the humanities, while the least intensity states - by the "high-reflecting" students of the humanities. The students of the humanities possessing either low or high level of self-reflection most often suffer from asthenical syndromes, whereas the "low-reflecting" engineering students demonstrate mental states of high arousal (liveliness, agitation). The states with medium arousal (commitment, calmness) are typical for both "medium-" and "high-reflecting" students of the technical sciences.

The analysis findings concerning the aggregated impact of the self-reflection's parameters and types of academic activity on the mental states revealed that during the lecture, in continuum from low level of the self-reflection to high one, the intensity of the mental states increases. As for the seminar and exam, the situation differs: the indicators of experienced mental states decline affected by the self-reflection. It should be noted that the states with high level of arousal are experienced by the students during the seminar, while the low level of arousal prevails at the exam. Thuswise, the lecture is the most favorable type of academic activity for the "high-reflecting" students (judging from the indicators of the mental stats' intensity), while the seminar is the same for the "low-reflecting" ones. The exam is the "stressful" type of academic activity for all students, despite the level of self- reflection.

\section{Conclusion}

The obtained results allow to assert the influence of self-reflection's level together with its leading constituent upon the mental states of the students in course of their academic activity. The self-reflection's influence upon these states is 
mediated by both the control abilities of the students, the type of academic activity and field of study, and the parameters of the mental states themselves.

\section{Findings}

1. The self-reflection's influence patterns upon the students' mental states differentiated on various criteria, such as modality, duration, sign and level of arousal were fixed. The self-reflection's influence upon the states with various modality is mediated by the tension of the academic activity: the high level of self-reflection in everyday situation makes an influence upon the volitional states, though in a stressful situation, vice versa, the intensity of volitional states increases, while decreases as for the emotional ones. It was also reported that the raise of self-reflection level cause the increase in transient states' intensity, while the expressiveness of the permanent ones falls down.

2. The states peculiar to everyday academic activity are felt most intensively in case of medium level of the cognitive self-reflection and high rates of the control one. The leading constituents of the mental states are the "behavioral" parameters. In course of stressful academic activity the "high self-reflecting" students more often feel the low intensity mental states, while the "low self-reflecting" ones evenly often feel both high and low intensity mental states.

3. The self-reflection's influence upon the mental states is mediated by the field of study, type of academic activity, and level of the students' control abilities: the raised levels of arousal are typical for "medium selfreflecting" students of humanities and "low self-reflecting" persons studying technical sciences. It was also stated that the most intensive mental states are experienced by the persons demonstrating equal characteristics of both the control abilities and self-reflection.

\section{Acknowledgements}

The article is written with funding from the Russian Foundation for Basic Research (RFBR) at the expense of Project No. 12-06-00043a.

\section{References}

Karpov, A.V., 2004. Psychology of Reflexive Mechanisms in Activity. Moscow: the Russian Academy of Sciences, the Institute of Psychology Press, pp: 450.

Farber, B.A., 1989. Psychological-Mindedness: Can There Be Too Much of a Good Thing? Psychotherapy: Theory, Research, Practice, Training, 26: 210-217.

Hua, W., M. Beitel, Z. Schuman-Oliver and D. Barry, 2007. Psychometric Properties of Chinese Version of the Psychological Mindedness Scale. Journal of the American Psychoanalytic Association, 55(1): 300-305.

Flavell, J.H., 1976. Metacognitive Aspects of Problem Solving. Resnick, L. B. (Ed.) The Nature of Intelligence. New York: Hillsdale (N. J.): Lawrence Erlbaum, pp: 231-135.

Astington, J.W.,1998. Theory of Mind, Humpty Dumpty, and the Icebox. Human development, 41: 30-39.

Premack, D. and G. Woodruff, 1978. Does the Chimpanzee Have a Theory of Mind? Behavioral and Brain Sciences, (1): 515-526.

Wegner, D.M.,2005. Who is the Controller of Controlled Processes?InThe New Unconscious, Eds.,Hassin, R.R., J.S. Uleman and J.A. Bargh. New York: Oxford University Press, pp: 19-36.

Prokhorov, A.O. and A.V. Chernov, 2012. Mental States Reflexive Control. Eds., Prokhorov, A.O. and A.V. Chernov. Bulletin of the Kazan University. Series: Humanities, Vol.154, Book 6: 244-257.

Karpov, A.V. and I.M. Skityayeva, 2005. Psychology of Personal Meta-Cognitive Processes. Eds., Karpov, A.V. and I.M.Skityayeva. Moscow: The Russian Academy of Sciences, the Institute of Psychology Press, pp: 352.

Grant, A.M., 2001. Rethinking Psychological Mindedness: Metacognition, Self-Reflection, and Insight. Behaviour Change,18: 8-17.

Morsanova, V.I. and E.M. Konoz, 2000. Styles of Self-Control in Human Behavior. Eds., Morsanova, V.I. and E.M.Konoz. Psychologylssues, 2: 118-127.

Schraw, G.and R.S. Dennison, 1994. Assessing Metacognitive Awareness. Contemporary Educational Psychology, 19: 460-475.

Nikiforov, G.S., 1988. Human Self-Control. Leningrad: The Leningrad State University Press, pp: 192.

Personal Mental States Diagnostics and Assessment Techniques, 2004. Eds.,Prokhorov, A.O. Moscow: PER SE Publishing,pp: 142147.

Sergiyenko, E.A., G.A. Vilenskaya and Yu.V. Kovaleva, 2011. Behavioral Control by the Subject. Eds., Sergiyenko, E.A., G.A.Vilenskaya and Yu.V.Kovaleva. Moscow: The Russian Academy of Sciences, the Institute of Psychology Press,pp: 353.

Prokhorov, A.O., 2005. Functional Structures and Means of Self-Control for Mental States. The Psychological Journal, Vol.26, 2: 68-80. 